\title{
I like my dog, does my dog like me?
}

Rehn, Therese; Lindholm, Ulrika; Keeling, Linda; Forkman, Björn

Published in:

Applied Animal Behaviour Science

DOI:

10.1016/j.applanim.2013.10.008

Publication date:

2014

Document version

Early version, also known as pre-print

Citation for published version (APA):

Rehn, T., Lindholm, U., Keeling, L., \& Forkman, B. (2014). I like my dog, does my dog like me? Applied Animal Behaviour Science, 150, 65-73. https://doi.org/10.1016/j.applanim.2013.10.008 


\title{
I like my dog, does my dog like me?
}

\author{
Therese Rehn ${ }^{\mathrm{a}, *}$, Ulrika Lindholm ${ }^{\mathrm{b}}$, Linda Keeling ${ }^{\mathrm{a}}$, Björn Forkman ${ }^{\mathrm{b}}$ \\ a Department of Animal Environment and Health, Swedish University of Agricultural Sciences, Box 7068, SE-750 07 Uppsala, Sweden \\ b Department of Large Animal Sciences, Faculty of Health and Medical Sciences, University of Copenhagen, Grønnegárdsvej 8,1870 \\ Frederiksberg C, Denmark
}

\section{A R T I C L E I N F O}

\section{Article history:}

Accepted 17 October 2013

Available online 27 October 2013

\section{Keywords:}

Canine

Human-animal relationship

MDORS

Strange Situation Procedure

Behaviour

Animal welfare

\begin{abstract}
A B S T R A C T
In this study, the possibility of there being an association between how an owner perceives his/her relationship to their dog and the way the dog experiences the relationship to its owner was investigated using two well-established methods within the anthrozoology literature. Twenty dog-owner dyads participated in the Strange Situation Procedure (SSP), to evaluate the bond on the dog's part, and the Monash Dog Owner Relationship Scale (MDORS) was used to investigate the strength of the relationship from the owner's view. Six attachment variables were created based on changes in the dogs' behaviour when it was with the owner vs. when it was with the stranger in the SSP. These were: changes in exploration, passive behaviour, independent play, social play, physical contact and tail wagging. The magnitude of these changes in the dogs' behaviour was then tested for correlations with the owners' scores in the MDORS. Only two correlations were found and both were with the MDORS subscale that measures "Dog-owner interaction". They suggested that owners who interact more frequently with their dog, have dogs showing more proximity-seeking behaviour upon reunion $(\rho=0.56, P=0.01, N=20)$ and less independent play behaviour $(\rho=-0.52, P=0.02, N=20)$. This might be a consequence of dogs being positively reinforced for close interaction by the owner, or these dogs may have developed an attachment style similar to insecurely attached children. No correlation was found between any of the six attachment variables in the SSP and the overall MDORS score, the MDORS subscale "Perceived costs" and, more importantly, no correlation to "Perceived emotional closeness". In summary, there is no support from this study for the view that the strength of the relationship an owner feels to his/her dog is mirrored in the strength of the bond of the dog to its owner.
\end{abstract}

(C) 2013 Elsevier B.V. All rights reserved.

\section{Introduction}

There is an increasing interest in factors associated with the quality of the dog-owner relationship and how relationships may vary between different dog-owner dyads (e.g. Hart, 1995; Kotrschal et al., 2009; Topál et al., 1998; Wedl et al., 2010). Most previous studies on the dog-owner

\footnotetext{
* Corresponding author at: Department of Animal Environment and Health, Swedish University of Agricultural Sciences, Box 7068, SE-756 51 Uppsala, Sweden. Tel.: +46 18672108; fax: +46 18673588 .

E-mail address: Therese.Rehn@slu.se (T. Rehn).
}

relationship rely on questionnaire studies investigating humans' attitudes towards dogs or correlations between the owner's personality/attachment pattern and his/her affectional bond with the dog. Based on such questionnaires, the relationship has been described in several ways, ranging from looking upon it like a master-slave relationship, to considering the dog as part of the family or saying it constitutes a child-parent bond (Barker and Barker, 1988; Sable, 2013; Serpell, 1995). It has also been reported that the presence of a pet dog may function as social support during performance of a stressful task (Allen et al., 1991; Beetz et al., 2011) and even as a substitute for human support and a source of comfort when emotionally distressed 
(Kurdek, 2009). Dogs are also suggested to act as social facilitators, in that owners feel more confident to engage in social interactions with other humans when accompanied by their dog (Messent, 1985) or due to the fact that dogs may attract other people to initiate a conversation with the owner (Guéguen and Ciccotti, 2008). Other studies have shown that owners provide a sense of security for their dogs and act as a buffer against stress (Gácsi et al., 2013) which may be even stronger than that of the presence of a companion dog (Tuber et al., 1996). These findings indicate a strong emotional bond between dogs and humans and vice versa.

Most owners live together with their pet dogs for a long time, allowing the formation of a stable (long-term) relationship. Human research has shown, that during the formation and continuation of stable social relationships, the behaviour of each individual in the dyad affects the types (or expectations) of future interactions (Berscheid and Peplau, 1983) as well as the quality of the relationship (Hinde, 1976). In order to describe properties of a relationship between two people however, it is important to consider each individual's perception or experience of the relationship, since these may differ between the two individuals (Hinde et al., 2001). In dog-human literature, effects of specific characteristics in the owner's personality on the relationship with their dog have been studied. For example, owners scoring high on a neuroticism scale report that they are strongly attached to their dogs (Kotrschal et al., 2009). There is also evidence indicating that dogs are more socially attracted to owners who are more 'neurotic' (Wedl et al., 2010), as shown by the level of approach and proximity seeking behaviour by dogs in the so called 'picture viewing test'. The test is performed in a novel environment during which time the owner's attention is diverted to pictures on the walls while the dog's behaviour is observed. More 'neurotic' owners also seem to use more gestural and verbal commands while interacting with their dogs (Kis et al., 2012). Interestingly, as in close human relationships (Monotoya et al., 2008), there is some evidence that the personality of dogs and their owners are similar to each other, as perceived by the owner anyway (Turcsán et al., 2012). To our knowledge however, characteristics of both individuals within the same dyad have rarely been measured in order to evaluate particular dog-owner relationships, excepting the work of Wedl et al. (2010). It seems reasonable to assume that a dog belonging to an owner who has a positive view of their relationship, which probably leads to a higher frequency of positive interactions, also experiences a close relationship to its owner. Therefore, this study aimed to investigate whether the owner's perception of his/her relationship with the dog could be reflected in the dog's behaviour during an attachment test.

The Monash Dog Owner Relationship Scale (MDORS) is a multi-dimensional questionnaire developed to specifically investigate the dog-owner relationship from the owner's point of view (Dwyer et al., 2006). The MDORS questionnaire consists of 28 questions, the answers of which contribute to an evaluation of the relationship according to three characteristics (subscales): dog-owner interaction (subscale 1), perceived emotional closeness (subscale 2) and perceived costs (subscale 3 ). The questionnaire has been validated and tested for reliability, resulting in Cronbach's coefficient alphas of $0.67,0.84$ and $0.84(N=1125)$ for subscales 1-3 (for details see Dwyer, 2004). It has been used in other studies investigating the dog-owner relationship with respect to, e.g. the occurrence of behavioural problems (Bennett and Rohlf, 2007), endocrine responses during interaction between owner and dog (Handlin et al., 2012) and responsible dog management among owners (Rohlf et al., 2010).

The Ainsworth's Strange Situation Procedure (ASSP) was developed within human developmental psychology to investigate attachment between toddlers and their parents (Ainsworth and Bell, 1970). The test includes challenging events aimed to activate the attachment system, an innate and adaptive motivational system that predisposes infants to seek the proximity of their attachment figure in times of distress (Bowlby, 1958). The balance between

Table 1

Information about breed, sex and age (years) of the participating dogs, gender of their owners as well as the length of their relationship (in months).

\begin{tabular}{|c|c|c|c|c|}
\hline Breed & Sex & Age (years) & Owner's gender & Length of relationship (months) \\
\hline Australian Kelpie & Male & 3.0 & Woman & 34 \\
\hline Belgian Malinois & Male & 2.5 & Woman & 29 \\
\hline Border Terrier & Neutered male & 6.0 & Woman & 69 \\
\hline Cane Corso & Female & 4.0 & Woman & 23 \\
\hline Cross bred & Neutered female & 2.5 & Woman & 12 \\
\hline Dalmatian & Neutered male & 7.0 & Man & 80 \\
\hline Dalmatian & Female & 2.5 & Woman & 26 \\
\hline German Shepherd & Male & 2.0 & Man & 21 \\
\hline German Shepherd & Male & 4.0 & Woman & 45 \\
\hline German Shepherd & Male & 4.5 & Man & 53 \\
\hline German Shepherd & Female & 4.5 & Woman & 52 \\
\hline German Shepherd & Female & 4.0 & Woman & 14 \\
\hline German Shepherd & Male & 4.5 & Man & 50 \\
\hline Labrador Retriever & Male & 4.5 & Woman & 54 \\
\hline Miniature Poodle & Female & 4.0 & Woman & 20 \\
\hline Miniature Schnauzer & Female & 4.0 & Woman & 14 \\
\hline Norwegian Lundehund & Male & 2.0 & Woman & 22 \\
\hline Petit Basset Griffon Vendéen & Male & 5.5 & Woman & 49 \\
\hline Tibetan Spaniel & Male & 5.0 & Woman & 58 \\
\hline Toy Poodle & Female & 8.5 & Woman & 100 \\
\hline
\end{tabular}




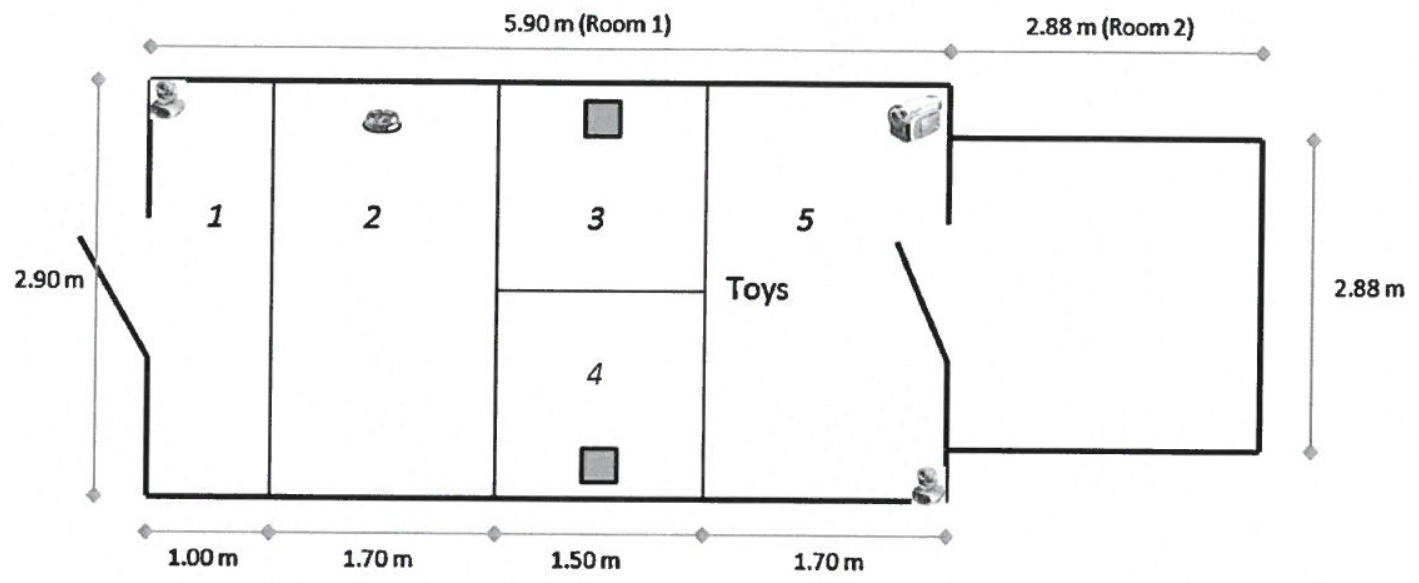

Fig. 1. Overview of the test area. The black lines represent the tape on the floor that divided the larger room (room 1) into five zones (1: near entrance door, 2: neutral zone, 3 and 4: owner's zone and stranger's zone each containing a chair, 5: neutral zone containing three toys). The entrance door is at the left hand side of the figure and there is a door between room 1 and room 2 shown to the right in the picture. Room 2 was empty and was available to the dogs from episode 4.

comfort seeking by the toddler to their object of attachment and other more independent behaviours such as exploration (once comfort has been obtained) is measured in the ASSP and different styles of attachment have been defined according to the dynamics in these behaviours (Ainsworth et al., 1978). Because the dog-owner relationship is proposed to share some features of the child-parent bond (Topál et al., 1998), modified versions of the ASSP, generally referred to as the Strange Situation Procedure (SSP), are increasingly being used to assess the affectional bond between dogs and humans (e.g. Palestrini et al., 2005; Palmer and Custance, 2008; Prato Previde et al., 2003; Valsecchi et al., 2010). Compared to other types of tests related to the dog-owner relationship (e.g. the 'picture viewing test' in Wedl et al., 2010), the SSP not only measures the proximity-seeking behaviour of the dog, but also includes aspects related to the sense of security the owner might provide the dog, which is an essential part of attachment theory (Bowlby, 1958). In this study, we used the SSP to investigate the dog's affectional bond to its owner as a measure of the strength of the relationship from the dog's point of view.

To investigate in detail the possibility of there being an association between the owner's perception of his/her relationship to the dog and the level of attachment behaviours expressed by the dog, two methods, frequently used separately in previous studies, were applied to the dog-owner dyads in this study: (1) the owners completed the MDORS questionnaire allowing assessment of their perception of their relationship with their dog and (2) dogs and owners participated in the SSP in order to evaluate the dog's affectional bond to its owner. The results from these two methods were then used to investigate possible correlations between the dog's bond to its owner and the owner's view of their relationship. The prediction is that the stronger the relationship is perceived as being by the owner, the more strongly bonded also the dog is to its owner.

\section{Material and methods}

\subsection{Subjects}

Twenty dog-owner dyads, recruited by advertisement and personal contacts, participated in the study (Table 1). The mean age of the owners was $34.6 \pm 3.4$ years $( \pm \mathrm{SE})$, ranging from 17 to 69 years old, and there were 16 women and four men. The mean age of the dogs was $4.1 \pm 0.4$ years and there were 12 males and eight females. All dogs were primarily kept as companion animals. The inclusion criteria were that the dog was between 2 and 8 years old and had been living together with the current owner for at least 6 months.

Before the study, dog owners were informed that the experiment was about the relationship between dogs and humans, but no specific details about the aim of the study were given. The study was reviewed and approved by the Swedish Ethical Committee on animal research in Uppsala, Sweden (Permit Number: C132/9) and by the Swedish Board of Agriculture for the use of privately owned dogs in research (Permit Number: 31-49977/09).

\subsection{MDORS questionnaire}

Before the SSP, the owner was asked to answer the MDORS questionnaire (for details, see Dwyer et al., 2006). The questions had been translated into Swedish. Although the questions were presented in a random order, they contribute to three subscales: the first subscale includes information about interactions between the dog and the owner (e.g. 'How often do you hug your dog?'); the second subscale deals with the emotional relationship with the dog (e.g. 'I wish my dog and I never had to be apart') and the last subscale is about costs and efforts of owning and caring for the dog (e.g. 'My dog costs too much money'). The answers in subscales 1 and 2 were labelled 1-5 (very seldom/totally disagree - very often/strongly agree) and the answers in 
Table 2

Episode overview of the protocol used in the test where the dog (D), the owner (O) and a stranger (S) participated (for further details see Rehn et al., 2013).

\begin{tabular}{|c|c|c|c|}
\hline Episode & Minute & $\begin{array}{l}\text { Present in the } \\
\text { test area }\end{array}$ & Main event in each episode \\
\hline 1 & $1 \mathrm{st}-3 \mathrm{rd}$ & $\mathrm{D}+\mathrm{O}$ & O sits quietly in the chair (ignoring the dog). \\
\hline 2 & 4th-6th & $\mathrm{D}+\mathrm{O}+\mathrm{S}$ & $\begin{array}{l}\text { S enters, sits quietly in chair for } 1 \text { min (ignoring the dog), starts a conversation with } O \text { for the } \\
\text { second min, then sits on floor and initiates play }{ }^{d} \text { with the dog using the toys during the last } \\
\text { min. Returns to chair after } 45 \mathrm{~s} \text { if dog does not want to play. O leaves the room unobtrusively } \\
\text { at the end of the episode. }\end{array}$ \\
\hline 3 & 7th-9th & $\mathrm{D}+\mathrm{S}$ & $\begin{array}{l}S \text { continues play/initiates play again with the dog. Returns to chair after } 45 \mathrm{~s} \text { if dog does not } \\
\text { want to play. } 20 \mathrm{~s} \text { before end of episode, } S \text { opens the door to room } 2 \text { and then leaves via the } \\
\text { entrance door in room } 1 \text {. }\end{array}$ \\
\hline 4 & 10th-12th & D & Dog is alone in test room, with access to room 2. \\
\hline 5 & 13th-15th & $\mathrm{D}+\mathrm{O}$ & 0 enters, waits $7 \mathrm{~s}$, greets the dog for $10 \mathrm{~s}$, then sits down and ignores the dog. \\
\hline 6 & 16th-18th & $D+S$ & $\begin{array}{l}\text { S enters, waits } 7 \mathrm{~s} \text {, greets the dog for } 10 \mathrm{~s} \text {, then sits down and ignores the dog. } 0 \text { leaves the } \\
\text { room when } S \text { stops greeting the dog. }\end{array}$ \\
\hline
\end{tabular}

a Before the test, the owner of the dog was shown the three toys and asked to 'grade' them according to what they believed would be most/least interesting/fun to the dog. The toy the owner thought was the most interesting to the dog was always introduced first by the stranger (initiating play for a maximum of $15 \mathrm{~s}$ per toy) and as soon as the dog was engaged in play, that toy was used. If the dog lost interest at any time, another toy was used. This gave a maximum time of $45 \mathrm{~s}(3 \times 15 \mathrm{~s})$ to initiate play with a dog that was not interested at all, or the dog could choose to play until the end of the episode with one or more toys.

subscale 3 were labelled 5-1 (very seldom/disagree - very often/strongly agree). The sum of scores from the total MDORS as well as for all questions within each subscale was calculated for the owner, where a high score represented a 'strong' bond in all subscales.

\subsection{The Strange Situation Procedure (SSP)}

Owners who participated in the SSP together with their dog were introduced to the protocol of the test and informed about how to behave before the experiment by a test leader who was not taking part in the SSP. During the actual test, owners were continuously guided by the female experimenter acting as the stranger. It was always the same person taking the role as the stranger and she had never met the dog before the test. The stranger wore the same clothes on all test occasions. The test leader was always situated in a room adjacent to the test area, monitoring the experiment.

\subsection{Test area for the SSP}

The test area comprised two relatively bare rooms separated by a door (Fig. 1). Room 1 was $17.1 \mathrm{~m}^{2}$ and room 2 was $8.3 \mathrm{~m}^{2}$. In the beginning of the test, the door between the two rooms was closed. Room 1 was divided into five areas using tape on the floor to facilitate monitoring of the dog's location during the test. The room included a water bowl, two chairs (one was allocated for the owner and the other one for the stranger, balanced between dyads throughout the experiment) and three toys (a ball, a rope tug toy and a squeaking toy).

One digital video camera: SONY Handycam ${ }^{\circledR}$ (HDRSR10E, Sony Corporation, Japan) and two wireless surveillance cameras (Vivotek network camera PT3124, VIVOTEK INC., Taiwan) covered room 1 . The test area was cleaned and disinfected between tests.

\subsection{Procedure}

The test consisted of six episodes each lasting for 3 min (Table 2). Reunion behaviour is suggested to be the most important aspect to consider when assessing attachment in toddlers (Ainsworth et al., 1978) and reunion events outside the test procedure are used when evaluating attachment in older children (Main and Cassidy, 1988). Therefore, we deliberately chose to use a similar protocol to that used by Palmer and Custance (2008), where there was a 'pure' reunion with the owner, i.e. the owner was the first one to come back to the room following the episode where the dog was completely alone. This is in contrast to some of the other SSP protocols where it is the stranger who is the first person to return after the dog has been alone.

\subsection{Data collection}

The videos were analyzed in real-time by two trained observers recording a total of 13 behavioural variables (Table 3), using instantaneous sampling every $5 \mathrm{~s}$ and one/zero sampling within the same time interval. These were summarized as proportion of time per episode or min, depending on the behaviour of interest and the type of comparison. Greeting behaviour, which measured the initial reaction of the dog to a person's return, was recorded both quantitatively (duration of tail wagging and physical contact) and qualitatively (scored $0-2$ from no greeting to intense greeting, adapted from Prato Previde et al., 2003).

\subsection{Statistical analyses}

Inter-observer agreement was analyzed using the Attribute Agreement Analysis in Minitab ${ }^{\circledR}$ Statistical Software (version 16.1.0, Copyright @ 2010 , Mintab Inc., USA) by means of parallel coding of $10 \%$ of the total sample (two video recorded tests). Inter-observer agreement was always higher than $88 \%$ (measured for each behaviour variable). All other analyses were performed in SAS ${ }^{\circledR}$ (version 9.2, Copyright (C) 2002-2010, SAS Institute Inc., Cary, NC, USA). 
Table 3

Ethogram.

\begin{tabular}{|c|c|c|}
\hline Behaviour & Definition & Sampling method \\
\hline Exploration & $\begin{array}{l}\text { Motor activity directed towards the physical aspects of the environment; the dog is } \\
\text { sniffing/licking/manipulating something within the distance of } 10 \mathrm{~cm} \text { (nose-object) }\end{array}$ & Instantaneous ( $5 \mathrm{~s}$ ) \\
\hline Passive & $\begin{array}{l}\text { Sitting, lying or standing without engaging in play, exploration and without being } \\
\text { focused on the door, stranger or owner }\end{array}$ & Instantaneous (5s) \\
\hline Social play & Motor activity directed towards a toy when interacting with a person & Instantaneous (5s) \\
\hline Independent play & Motor activity directed towards a toy without any interactions with a person & Instantaneous (5s) \\
\hline Following & $\begin{array}{l}\text { Locomotion directed against/behind/in the same direction as a person }{ }^{\text {a }} \text { who is also in } \\
\text { locomotion }\end{array}$ & $1 / 0$ sampling $(5 s)$ \\
\hline Oriented to door & Sitting, standing or lying and focused on the entrance door $(>2 s)$ & $1 / 0$ sampling $(5 \mathrm{~s})$ \\
\hline Oriented to person & Sitting, standing or lying and focused on person ${ }^{\mathrm{a}}(>2 \mathrm{~s})$ & $1 / 0$ sampling $(5 s)$ \\
\hline Contact door & $\begin{array}{l}\text { Scratching, jumping up against the door and/or physical contact with the door with } \\
\text { the muzzle }\end{array}$ & $1 / 0$ sampling $(5 \mathrm{~s})$ \\
\hline Contact person & Physical contact with person, ${ }^{\mathrm{d}}$ initiated by the dog, regardless of visual orientation & $1 / 0$ sampling $(5 s)$ \\
\hline Vocalization & Barking, growling, howling and/or whining & $1 / 0$ sampling $(5 \mathrm{~s})$ \\
\hline Location & Zone $1,2,3,4,5$, or room 2 & Instantaneous (5s) \\
\hline Tail wagging & Repetitive wagging movement of the tail & $1 / 0$ sampling $(5 \mathrm{~s})$ \\
\hline Greeting & Approaching, tail wagging, jumping and contact directed towards the entering person ${ }^{\mathrm{a}}$ & Score $0-2^{\mathrm{b}}$ \\
\hline
\end{tabular}

Wilcoxon signed rank tests were used to explore the dogs' reactions during the SSP. The dogs' behaviour towards, or in the presence of, the owner and the stranger was compared to each other to test whether the dogs used their owner as a secure base and if they discriminated between the owner and the stranger. The proportion of time spent performing exploration and independent play or in passive behaviour was compared between episode 5 , where the owner was present, and episode 6 , where only the stranger was present. The level of social play was compared using data collected from the last minute of episode 2 , when the owner was present, and the first minute of episode 3 , when only the stranger was present. Seeking the proximity of the owner or the stranger was compared by testing behaviour scores (oriented towards person, following person, located in person's zone and physical contact with person) collected in episodes 1 and 5 , where the owner was present, with scores recorded in episodes 3 and 6 (stranger present). The proportion of time in physical contact with person and tail wagging were compared by testing the scores collected during reunion with the owner in the first minute of episode 5 with those during reunion with the stranger in the first minute of episode 6 .

Six attachment variables were created based on differences in the dogs' behaviour between episodes of the SSP where the owner was either present (episodes 1,2 and 5) or not (episodes 3, 4 and 6). These were created using similar comparisons as described above, but with the aim to describe the magnitude of the change in behaviour of the dog as the difference of the duration, measured as a proportion of time, spent performing a particular behaviour in the two situations. For example, the variable 'change in exploration' was calculated by taking the difference between the time a dog spent exploring when accompanied by its owner and the time it spent exploring when the owner was absent. In this particular case, the hypothesis was that the stronger the bond to the owner, the more the dog would explore when the owner was present (using the owner as a secure base) compared to when the owner was absent; i.e. a large, positive value for the 'change in exploration' variable. Variable measures for 'change in exploration', 'change in independent play' and 'change in passive behaviour' were calculated for each dog based on data collected in episode 5 and episode 6. For 'change in social play', which was offered to the dog only during the last minute of episode 2 and the first minute of episode 3 , differences in the duration of play behaviour between these times were calculated. With regards to the reunion behaviours, 'change in physical contact' and 'change in tail wagging', variable measures were based on differences during the first minute of episode 5 , when the owner returned, and the first minute of episode 6 , when the stranger returned.

Each of the six attachment variables, as well as the length of a dyad's relationship (duration of ownership in months), were correlated with the total MDORS score, as well as the scores from each MDORS subscale, using Spearman's rank-order correlation tests.

\section{Results}

\subsection{Dogs' relationship to their owners (SSP)}

The results of the comparison of the dogs' behaviour when it was together with the owner vs. when the owner was absent and the dog was alone together with the stranger, were similar to those that have been found previously (e.g. Palmer and Custance, 2008; Prato Previde et al., 2003). The results from this study are summarized in Table 4.

The distributions of the magnitude of changes in dog's behaviour in those episodes when only the owner was present $v s$. those episodes when the owner was absent but where a stranger was together with the dog (attachment variables) are presented in Fig. 2 . These values were further used for the correlation tests in Section 3.3. 
Table 4

Summary of results from the dogs' behaviour during the Strange Situation Procedure. Comparisons of the dogs' behaviour were made between episodes where the owner was present (episodes (Ep) 1,2 and $5 ; \min 6,13$ ) and where the stranger was present (episodes (Ep) 2, 3 and 6; min 7, 16). Results are presented as mean proportion of time $\pm \mathrm{SE}$

\begin{tabular}{|c|c|c|c|}
\hline Behaviour & Comparison & & Results \\
\hline Secure-base effects & Ep 5 & Ep 6 & \\
\hline Exploration & $0.06 \pm 0.02$ & $0.003 \pm 0.002$ & $S(N=20)=26, P=0.006$ \\
\hline Passive & $0.43 \pm 0.02$ & $0.40 \pm 0.02$ & ns \\
\hline Independent play & $0.22 \pm 0.08$ & $0.11 \pm 0.06$ & $S(N=20)=18, P=0.008$ \\
\hline & Min 6 & Min 7 & \\
\hline Social play & $0.60 \pm 0.08$ & $0.41 \pm 0.10$ & $\mathrm{~S}(N=20)=46, P=0.03$ \\
\hline Proximity-seeking & Ep $1+5$ & Ep $3+6$ & \\
\hline Oriented to person & $0.07 \pm 0.02$ & $0.02 \pm 0.005$ & $S(N=20)=37.5, P=0.03$ \\
\hline Contact person & $0.07 \pm 0.02$ & $0.03 \pm 0.01$ & $S(N=20)=40.5, P=0.08$ \\
\hline Following person & $0.01 \pm 0.02$ & $0.03 \pm 0.03$ & $S(N=20)=-69, P=0.004$ \\
\hline Vocalization & $0.09 \pm 0.03$ & $0.23 \pm 0.06$ & $S(N=20)=-56, P=0.006$ \\
\hline Contact door & $0.01 \pm 0.004$ & $0.02 \pm 0.007$ & $S(N=20)=-19.5, P=0.02$ \\
\hline Location near door & $0.08 \pm 0.02$ & $0.34 \pm 0.06$ & $S(N=20)=-78.5, P<0.001$ \\
\hline Oriented to door & $0.06 \pm 0.02$ & $0.27 \pm 0.05$ & $\mathrm{~S}(N=20)=-72.5, P<0.001$ \\
\hline Greeting & Min 13 & Min 16 & \\
\hline Greeting score & $1.35 \pm 0.11$ & $0.85 \pm 0.11$ & $S(N=20)=18, P=0.008$ \\
\hline Physical contact person & $0.30 \pm 0.06$ & $0.07 \pm 0.02$ & $\mathrm{~S}(N=20)=59, P<0.001$ \\
\hline Tail wagging & $0.72 \pm 0.05$ & $0.42 \pm 0.07$ & $S(N=20)=91.5, P<0.001$ \\
\hline
\end{tabular}

a) Changes in exploration

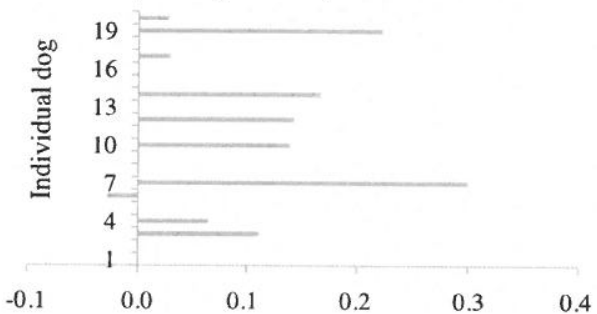

c) Changes in independent play

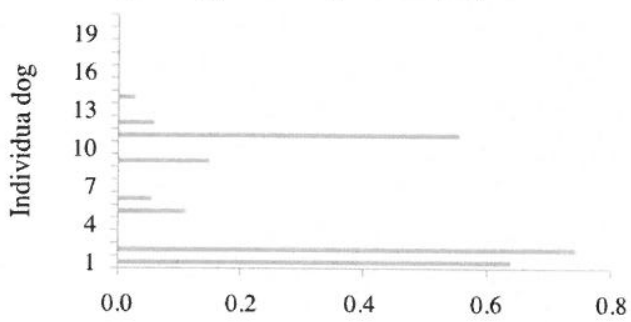

e) Changes in physical contact with person during greeting

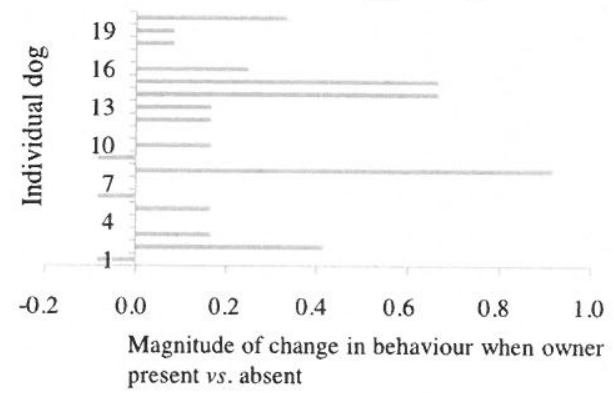

b) Changes in social play

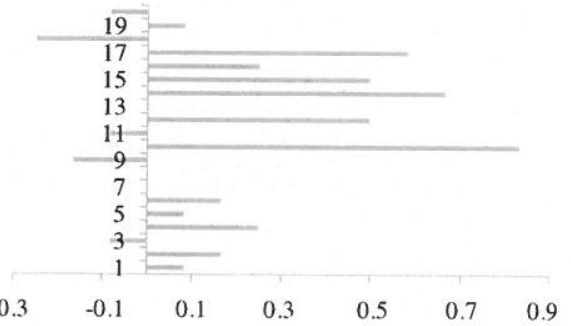

d) Changes in passive behaviour

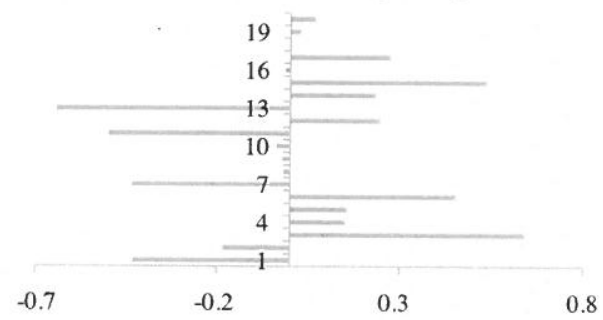

f) Changes in tail wagging during greeting

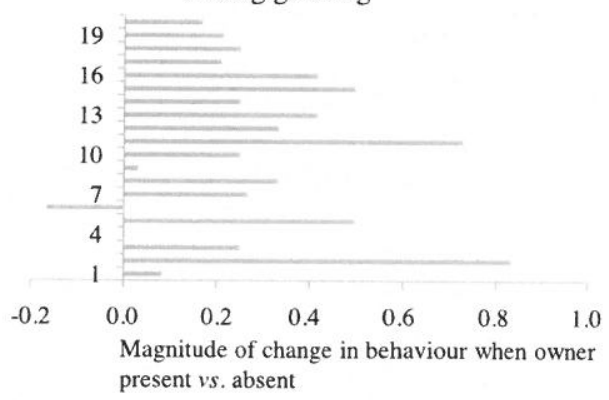

Fig. 2. Distribution of the magnitude of changes ( $x$-axis, proportion of time) when owner present vs. owner absent ( $\Delta$-values) in the dogs' ( $y$-axis) levels of (a) exploratory behaviour (episode 5 vs. 6), (b) social play (min 6 vs. 7), (c) individual play (episode 5 vs. 6) and (d) passive behaviour (episode 5 vs. 6). The figure also presents changes in the dogs' levels of (e) initiating physical contact with person (min 13 vs. 16) and (f) tail wagging (min 13 vs. 16) when greeting the owner $v$ s. the stranger ( $\Delta$-values). 


\subsection{Owners' relationship to their dogs (MDORS)}

The scores achieved in the questionnaire were summarized to a total MDORS score as well as separately for each MDORS subscale for each dog owner. In our sample, the owners' scored a total of between 93 and 122 points $(109.7 \pm 1.7$, mean $\pm S E)$ for the total MDORS score, out of a potential range from 28 to 140 points. In subscale 1 (dog-owner interaction) the owners' scores ranged between 30 and 39 points $(34.7 \pm 0.6)$ out of a possible range from 9 to 45 points. For subscale 2 (perceived emotional closeness) the owners scored between 30 and 47 points $(36.4 \pm 1.0)$ out of a range from 10 to 50 points. In subscale 3 (perceived costs), the owners scored between 30 and 45 points $(37.9 \pm 1.1)$ out of a range from 9 to 45 points. The total MDORS score for each owner, as well as their scores in each subscale were used for further correlation tests in Section 3.3.

\subsection{Correlations between SSP and MDORS}

Since we did not observe a difference in the level of passive behaviour according to which person was accompanying the dog within the SSP, possible correlations between the variable 'changes in passive behaviour' and MDORS results were never tested.

All results from the Spearman rank-order correlation analyses are presented in Table 5. Only two significant correlations were found, both involving MDORS subscale 1 (dog-owner interaction). The attachment variable 'change in independent play' was negatively correlated with subscale 1 and there was a positive correlation between subscale 1 and the 'change in physical contact' with the owner and with the stranger at reunion. There was no significant correlation between the length of the dyad's relationship (Table 1) and the MDORS.

\section{Discussion}

The characteristics of both the owner and the dog are rarely combined when evaluating the quality of a particular dog-human relationship. Therefore, in this study we wanted to test whether or not there were any associations between how the owner perceived the relationship with their dog and the strength of the dog's bond to its owner. Results showed that dogs who initiated more physical contact with their owners upon reunion during the SSP had owners who scored high in MDORS subscale 1, i.e. were owners who interacted a lot with their dogs. Furthermore, a negative correlation was found between changes in independent play in the SSP and MDORS subscale 1, i.e. dogs belonging to owners that interacted a lot with them on a regular basis played less on their own. There was no correlation to the overall MDORS, no correlation to the subscale "Perceived cost" and, perhaps most importantly, we found no correlations between the dog's behaviour in the SSP test and the MDORS subscale "Perceived emotional closeness". In summary, there is no support from this study for the view that the strength of the relationship an owner feels to his/her dog is mirrored in the strength of the relationship of the dog to its owner.
The fact that owners who interact a lot with their dog on a daily basis (playing, rewarding, and having physical interactions, etc. with the dog) had dogs who initiated much more physical contact upon reunion with their owner may be explained by the previous experience of the dog, where it has been rewarded by the owner for initiating physical contact. Also, based on the items included in MDORS subscale 1, these owners spend more time with their dog, e.g. in front of the TV and when visiting friends. So the increased physical contact at reunion may be merely a reflection of a more owner-dependent dog who is not as used to being left alone and hence reacts by showing increased comfortseeking behaviour during this challenging test situation. This interpretation somewhat supports the results by Wedl et al. (2010) who found a positive correlation between the amount of time the owner spent with their dog and the level of the dog's social attraction to the owner.

The dogs who initiated a lot of physical contact with their owners upon reunion also showed least difference in play when in the presence of their owner vs. the stranger, indicating that they did not use their owner as a secure base from whom to move away and confidently engage in more independent behaviour. One could speculate that the behaviour pattern in our study reflects features of an attachment style resembling the 'clinging' behaviour of children with an insecure ambivalent attachment style (e.g. Ainsworth et al., 1978; Main and Solomon, 1986). Noteworthy though, was that none of the owners in this study reported having a dog with behavioural problems related to separation anxiety, so these patterns seem to be subtle outcomes of their routine interactions. An alternative explanation may be that these dogs are so well socialized (as they are used to accompanying their owners) that they did not change their independent play behaviour based on whether or not the owner was present in the room. The latter would then resemble an insecure avoidant attachment style.

The dogs in this study were mainly pure-bred, coming from a specific region of Sweden and most owners were women. As the participation was voluntary, there is always the risk of attracting a specific type of dog owner, which may also have biased the test population. These facts, in addition to the rather limited number of subjects, means that the results including the absence of other significant correlations, should be interpreted cautiously. It may be that a larger, more heterogeneous sample would have revealed more aspects related to the dynamics in the dog-owner relationship. Although the results of this study do imply that these correlations, if they exist, are weak. However, the approach used in this study raises a variety of issues about quantifying the strength of the relationship that are discussed in more detail below.

\subsection{Using the SSP and MDORS as test tools}

Several aspects of the dogs' behaviour during the SSP in this study were in agreement with attachment theory and support findings in previous studies (e.g. Palmer and Custance, 2008; Prato Previde et al., 2003; Topál et al., 1998). Dogs explored and played more in the presence of their owner and they showed more proximity-seeking 
Table 5

Summary of the results from the Spearman rank correlation tests between changes of the dog's behaviour in the Strange Situation Procedure (SSP) and owner's score in the Monash Dog Owner Relationship Scale (MDORS) total, subscale 1, subscale 2 and subscale 3 (spearman rho) ( $N=20$ ).

\begin{tabular}{|c|c|c|c|c|}
\hline Behavioural variable in SSP & MDORS total & MDORS subscale 1 & MDORS subscale 2 & MDORS subscale 3 \\
\hline Change in exploration & -0.36 & -0.08 & -0.26 & -0.14 \\
\hline Change in social play & -0.24 & 0.05 & 0.07 & -0.35 \\
\hline Change in independent play & 0.08 & -0.52 & 0.35 & 0.02 \\
\hline Change in physical contact at reunion & 0.24 & 0.56 & 0.16 & -0.13 \\
\hline Change in tail wagging at reunion & 0.36 & 0.27 & 0.30 & 0.23 \\
\hline Relationship length & 0.20 & 0.08 & 0.07 & 0.10 \\
\hline
\end{tabular}

behaviours and greeted their owner more intensively than the stranger. These results indicate that the owner is a unique person to the dog from whom it seeks comfort, security and reassurance.

The approach used to assess the strength of the affectional bond was based on assumptions within attachment theory. It was first assumed that differences in the amount of time spent performing key behaviours related to the secure base effect (exploration, play and passive behaviour) when the dog was with the owner compared to the stranger was related to the strength of the affectional bond. Although logical, this assumption might be wrong since it encompasses behaviours which are influenced not only by the quality of the bond, but also by dog personality (e.g. Marinelli et al., 2007; Svartberg et al., 2005). Another assumption was that differences in the amount of proximity-seeking behaviour reflect the strength of the bond. It seems reasonable to believe that these behaviours are less affected by factors such as personality or training of the dog and hence more related to an affectional bond.

As stated earlier, reunion behaviour has been suggested to be the most relevant indicator of attachment levels (Ainsworth et al., 1978; Marinelli et al., 2007; Topál et al., 1998). This implies that more detailed observations of the dogs' greeting responses would serve as a reliable indicator of the strength of the bond. One cannot however exclude that the higher levels of physical contact initiated by the dog upon reunion are merely a consequence of being rewarded for this behaviour previously. Although it may also be that rewarding the dog for close physical contact in the past has enhanced the bond.

The SSP has been frequently used to evaluate the bond between dogs and their owners, but the test has been criticized regarding general procedural problems (Wedl et al., 2010), but also more specific issues concerning possible order effects (Fallani et al., 2006; Palestrini et al., 2005; Palmer and Custance, 2008; Prato Previde et al., 2003). An evaluation of the SSP, including the usual treatment where dogs participated in the test with a familiar person and a stranger, as well as a control treatment where two strangers participated with the dogs, showed that exploration decreased throughout the test procedure irrespective of whether there was a familiar person in the room or a stranger who followed the same protocol as the familiar person (Rehn et al., 2013). In this study however, the aim was to look at differences in the magnitude of the change in behaviour, where all dogs were subjected to the same test set up, and to compare it with the owner's scores from the relationship questionnaire. This makes the study less sensitive to these specific problems. The SSP was originally developed to investigate the behavioural responses of very young children. Dogs differ in their background and their training and management (such as being separated from the owner in different contexts and environments) probably affects the results of the SSP to a large extent. At the same time, there are no other well recognized tests that assess the bond between animal and human, which left us with few alternative methods to use in the current study. Nevertheless, the limitations mentioned above might make the procedure less sensitive to the strength of the bond and so explain why few correlations were found in this study.

The behaviour of dogs during the SSP (related to the reunion episodes with the owner and the stranger) was only correlated to the owners' score in MDORS 'dog-owner interaction' subscale. Although seemingly logical to interpret, this subscale has the lowest reliability with a Cronbach's coefficient alpha at 0.67 (Dwyer, 2004; Dwyer et al., 2006). The main reason for this is that answers in this subscale may be influenced by the owner's lifestyle, e.g. whether or not the owner owns a car (How often do you take your dog in the car?) or how often he/she is visiting friends (How often do you take your dog to visit people?). Indeed how often the person is reunited with their dog and the length of time they are separated on each occasion will ultimately influence the type of reunion behaviour that develops between the dog and its owner.

In this study, dogs were assessed based on their reaction to an immediate stressor while owners were tested using their perception of a situation, rather than putting the owner in that situation and observing their reactions. Thus we used an attitudinal approach to assess the owner's bond while a behavioural test was used to evaluate the dog's bond. In the future, the reaction of both members in the dyad during a challenging situation could serve as a better measure of the strength of the relationship. In such a situation though, it is difficult to entangle whether or not social referencing influences the reactions, i.e. the fact that the behaviour of the owner could affect how the dog reacts, as has been shown to a frightening stimulus (Merola et al., 2012). Furthermore, the MDORS is not based on the specific criteria included in attachment theory (Dwyer et al., 2006), but only covers parts of it. In future studies, a questionnaire-based evaluation of the owner should perhaps be more closely linked to items related to attachment theory, so that the same types of motivational systems are investigated in both dyad members. There is clearly more work needed to investigate both sides of the dog-owner relationship. 


\section{Conclusion}

The findings in this study suggest that owners that interact frequently with their dog on a regular basis have dogs showing more proximity-seeking behaviour upon reunion and less independent play behaviour. This might be a consequence of being reinforced for close interaction by the owner, or these dogs may have developed an attachment style similar to insecurely attached children. Other than this there was no evidence to support the view that because a person has a strong emotional bond to their dog, their dog is similarly attached to them, but these results should be confirmed in a larger study.

\section{Acknowledgements}

We wish to thank all dogs and their owners for participating in this study. The study was funded by the Swedish Research Council for Environment, Agricultural Sciences and Spatial Planning. The final stages of this work were carried out within the Centre of Excellence in Animal Welfare Science, a Swedish collaborative research environment.

\section{References}

Ainsworth, M.D.S., Bell, S.M., 1970. Attachment, exploration and separation: Illustrated by the behavior of one-year-olds in a strange situation. Child Dev. 41, 49-67.

Ainsworth, M.D.S., Blehar, M.C., Waters, E., Wall, S., 1978. Measures and methods of attachment. In: Ainsworth, M.D.S., Wall, S. (Eds.), Patterns of Attachment: Psychological Study of the Strange Situation. L Eerbaum Ass. Inc., Hillsdale, New Jersey, pp. 45-64.

Allen, K.M., Blascovich, J., Tomaka, J., Kelsey, R.M., 1991. Presence of human friends and pet dogs as moderators of automatic responses to stress in women. J. Pers. Soc. Psychol. 61, 582-589.

Barker, S.B., Barker, R.T., 1988. The human-canine bond: closer than family ties? J. Ment. Health Couns. 10, 46-56.

Beetz, A., Kotrschal, K., Turner, D.C., Hediger, K., Uvnäs-Moberg, K., Julius, H., 2011. The effect of a real dog, toy dog and friendly person on insecurely attached children during a stressful task: an exploratory study. Anthrozoos 24, 349-368.

Bennett, P., Rohlf, V., 2007. Owner-companion dog interactions: relationships between demographic variables, potentially problematic behaviours, training engagement and shared activities. Appl. Anim. Behav. Sci. 102, 65-84

Berscheid, E., Peplau, L.A., 1983. The emerging science of relationships In: Kelley, H.H., Christensen, A., Harvey, J.H., Huston, T.L., Levinger, G McClintock, E., Peplau, L.A., Peterson, D.R. (Eds.), Close Relationships. W.H. Freeman and Company, New York, pp. 1-19.

Bowlby, J., 1958. The nature of the child's tie to his mother. Int. J. Psychoanal. 39, 350-373.

Dwyer, F., 2004. The Development of a Multi-Dimensional Human-Canine Relationship Questionnaire: The Monash Dog Owner Relationship Scale. Monash University, Clayton, Victoria, Australia, pp. 710 (Doctoral Thesis).

Dwyer, F., Bennett, P.C., Coleman, G.J., 2006. Development of the Monash Dog Owner Relationship Scale (MDORS). Anthrozoos 19, 243-256.

Fallani, G., Prato Previde, E., Valsecchi, P., 2006. Do disrupted early attachments affect the relationship between guide dogs and blind owners? Appl. Anim. Behav. Sci. 100, 241-257.

Gácsi, M., Maros, K., Sernkvist, S., Faragó, T., Miklósi, Á., 2013. Human analouge safe haven effect of the owner: behavioural and heart rate response to stressful social stimuli in dogs. PLoS ONE 8 (3), e58475 http://dx.doi.org/10.1371/journal.pone.0058475.

Guéguen, N., Ciccotti, S., 2008. Domestic dogs as facilitators in social interactions: an evaluation of helping and courtship behaviors. Anthrozoos 21, 339-349

Handlin, L., Nilsson, A., Ejdebäck, M., Hydbring-Sandberg, E., UvnäsMoberg, K., 2012. Associations between the psychological characteristics of the human-dog relationship and oxytocin and cortisol levels. Anthrozoos 25, 215-228.

Hart, L.A., 1995. Dogs as human companions: a review of the relationship. In: Serpell, J. (Ed.), The Domestic Dog, its Evolution, Behaviour and Interactions with People. Cambridge University Press, Cambridge, pp. $161-178$

Hinde, R.A., 1976. On describing relationships. J. Child Psychol. Psychiatry $17,1-19$.

Hinde, R.A., Finkenauer, C., Auhagen, A.E., 2001. Relationships and the selfconcept. Pers. Relatsh. 8, 187-204

Kis, A., Turcsán, B., Miklósi, Á., Gácsi, M., 2012. The effect of the owner's personality on the behaviour of owner-dog dyads. Interact. Studies 13, 371-383.

Kotrschal, K., Schöberl, I., Bauer, B., Thibeaut, A., Wedl, M., 2009. Dyadic relationships and operational performance of male and female owners. Behav. Processes 81, 383-391.

Kurdek, L.A., 2009. Pet dog as attachment figures for adult owners. J. Fam. Psychol. 23, 239-446.

Main, M., Cassidy. J., 1988. Categories of response to reunion with the parent at age 6: predictable from infant attachment classifications and stable over a 1-month period. Dev. Psychol. 24, 415-426.

Main, M., Solomon, J., 1986. Discovery of a nonsecure disoriented attachment pattern. In: Brazelton, T., Youngman, M. (Eds.), Affective Development in Infancy. Ablex Publishing, Westport, CT, pp. 95-142.

Marinelli, L., Adamelli, S., Normando, S., Bono, G., 2007. Quality of life of the pet dog: influences of owner and dog's characteristics. Appl. Anim. Behav. Sci. 108, 143-156.

Merola, I., Prato Previde, E., Marshall-Pescini, S., 2012. Dogs' social referencing towards owners and strangers. PLOS ONE 7 (10), e47653, http://dx.doi.org/10.1371/journal.pone.0047653.

Messent, P.R., 1985. Pets as social facilitators. Vet. Clin. North Am.: Small Anim. Pract. 15, 287-393.

Monotoya, R.M., Horton, R.S., Kirchner, J., 2008. Is actual similarity necessary for attraction? A meta-analysis of actual and perceived similarity. J. Soc. Pers. Relat. 25, 889-922.

Palestrini, C., Prato Previde, E., Spiezio, C., Verga, M., 2005. Heart rate and behavioural responses of dogs in the Ainsworth's Strange Situation: a pilot study. Appl. Anim. Behav. Sci. 94, 75-88.

Palmer, R., Custance, D., 2008. A counterbalanced version of Ainsworth's Strange Situation Procedure reveals secure-base effects in dog-human relationships. Appl. Anim. Behav. Sci. 109, 306-319.

Prato Previde, E., Custance, D.M., Spiezio, C., Sabatini, F., 2003. Is the dog-human relationship an attachment bond? An observational study using Ainsworth's strange situation. Behaviour 140, 225-254.

Rehn, T., McGowan, R.T.S., Keeling, L.J., 2013. Evaluating the Strange Situation Procedure (SSP) to assess the bond between dogs and humans. PLoS ONE 8 (2), e56938, http://dx.doi.org/10.1371/journal. pone.0056938

Rohif, V., Bennett, P., Toukhsati, S., Coleman, G., 2010. Why do even committed dog owners fail to comply with some responsible ownership practices? Anthrozoos 23, 143-155

Sable, P., 2013. The pet connection: an attachment perspective. Clin. Soc Work J. 41, 93-99.

Serpell, J.A., 1995. From paragon to pariah: some reflections on human attitudes to dogs. In: Serpell, J. (Ed.), The Domestic Dog, its Evolution, Behaviour and Interactions with People. Cambridge University Press, Cambridge, pp. 245-256.

Svartberg, K., Tapper, I., Temrin, H., Radesäter, T., Thorman, S., 2005. Consistency of personality traits in dogs. Anim. Behav. 69, 291-383.

Topál, J., Miklósi, Á., Csányi, V., Dóka, A., 1998. Attachment behavior in dogs (Canis familiaris): a new application of Ainsworth's (1969) Strange Situation Test. J. Comp. Psychol. 112, 219-229.

Tuber, D.S., sanders, S., Hennessy, M.B., Miller, J.A., 1996. Behavioral and glucocorticoid responses of adult domestic dogs (Canis familiaris) to companionship and social separation. J. Comp. Psychol. 110, 103-108.

Turcsán, B., Range, F., Virányi, Z., Miklósi, Á., Kubinyi, E., 2012. Birds of feather flock together? Perceived personality matching in owner-dog dyads. Appl. Anim. Behav. Sci. 140, 154-160.

Valsecchi, P., Prato Previde, E., Accorsi, P.E., Fallani, G., 2010. Development of the attachment bond in guide dogs. Appl. Anim. Behav. Sci. 123 43-50.

Wedl, M., Schöberl, I., Bauer, B., Day, J., Kotrschal, K., 2010. Relational factors affecting dog social attraction to human partners. Interact. Studies $11,482-503$. 